Supporting Information for

\title{
Aggregation Behavior of the Blends of Homo-PS and PS- $b$-PEO- $b$-PS at the Air/Water Interface
}

\author{
Wei Xu, Gangyao Wen*, Tao Wu, and Nanyang Chen \\ Department of Polymer Materials and Engineering, College of Material Science and \\ Engineering, Harbin University of Science and Technology, 4 Linyuan Road, Harbin \\ 150040, P. R. China
}

* Corresponding Author. E-mail: gywen@hrbust.edu.cn (G. Wen).

Website: http://wengangyao.polymer.cn/ (G. Wen). 


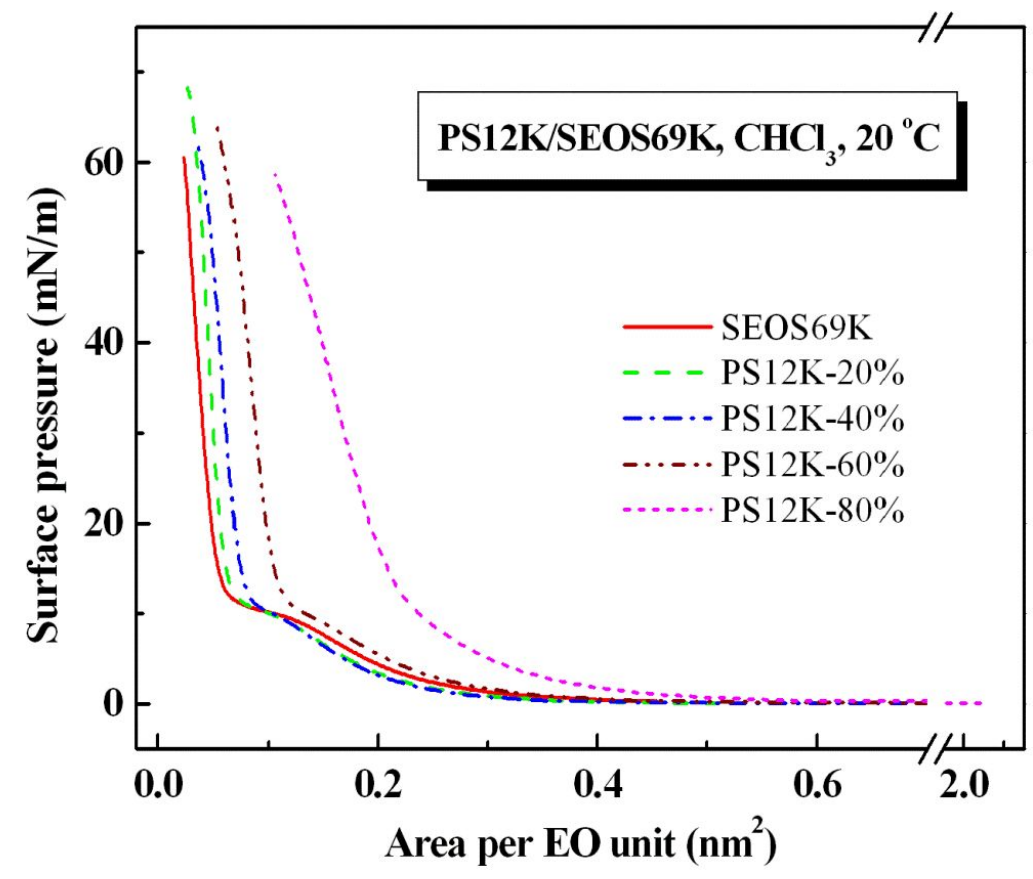

Figure S1. $\pi-A$ isotherms of the monolayers of pure SEOS69K and the PS12K/SEOS69K blends at $20^{\circ} \mathrm{C}$.

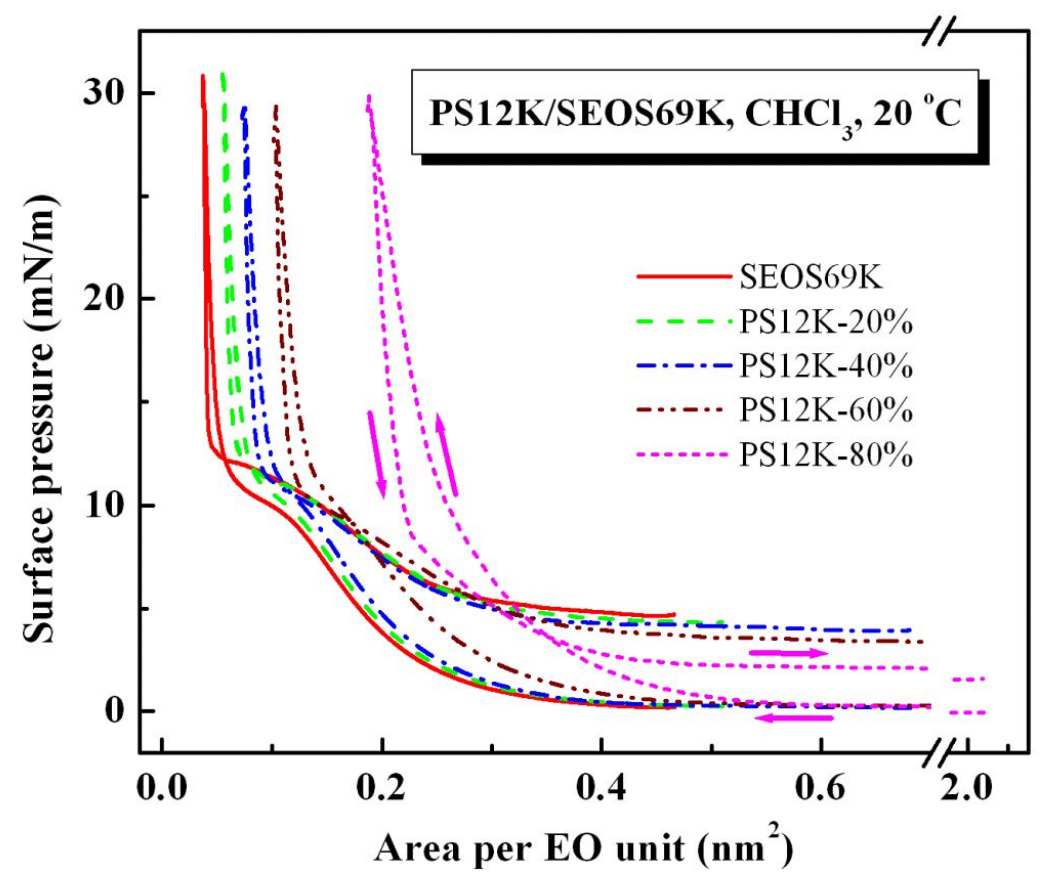

Figure S2. Hysteresis curves of the monolayers of pure SEOS69K and the PS12K/SEOS69K blends. The maximum pressure is $30 \mathrm{mN} / \mathrm{m}$. 